\title{
Comparative Enantioseparation of Amlodipine by HPLC and Capillary Electrophoresis
}

\author{
Anca Gabriela Cârje, Alina Balint*, Daniela-Lucia Muntean, Gabriel Hancu, Valentin Ion, Silvia Imre
}

University of Medicine and Pharmacy from Tirgu Mures, Romania

Objective: The purpose of this study was to separate the enantiomers of amlodipine by High Performance Liquid Chromatography (HPLC) using ovomucoid (OVM) as chiral selector, respectively by Capillary Electrophoresis (CE) using cyclodextrines and to evaluate the analytical performance of the both proposed methods. Material and methods: HPLC enantioseparation of amlodipine was performed on an HPLC Agilent Technologies 1100 series using as chiral stationary phase an Ultron ES OVM, 150x4.6 mm column with ovomucoid as chiral selector. The stereoselective CE analysis of amlodipine was achieved on Agilent Technologies $7100 \mathrm{CE}$ using uncoated fused-silica capillaries $48 \mathrm{~cm} \times$ $50 \mathrm{~mm}$ and different type of cyclodextrins as chiral selectors. Results: A mobile phase consisting of $80 \% \mathrm{Na} 2 \mathrm{HPO} 410 \mathrm{mM}$ at a pH level of 5.0 and $20 \% \mathrm{ACN}$, isocratic elution at a flow of $1 \mathrm{ml} / \mathrm{min}$ turned to be the optimal experimental conditions for HPLC analysis (R=5.51; $\alpha=1.71)$ with retention times shorter than 10 minutes for the two isomers, tR (S-AML) $=4.63(\mathrm{~min})$; tR (R-AML) $=5.54$ (min). The migration times for amlodipine enantiomers were $\mathrm{tm}(\mathrm{S}-\mathrm{AML})=8.15(\mathrm{~min})$ and $\mathrm{tm}(\mathrm{R}-\mathrm{AML})=8.45(\mathrm{~min})$ and the optimum $\mathrm{CE}$ conditions have proven to be a buffer solution containing $25 \mathrm{mM} \mathrm{H3PO} 4$ at $\mathrm{pH} 3.0$ and $20 \mathrm{mM} \alpha-\mathrm{CD}$ as chiral selector and a capillary temperature set at $15^{\circ} \mathrm{C}(\mathrm{R}=1.51 ; \alpha=1.03)$. Conclusion: The analytical performances of the chromatographic method using OVM as chiral selector are superior to the electrophoretic analysis method but the CE method is more economical and may represent an alternative to the HPLC chromatographic separation.

Keywords: amlodipine, enantioseparation, ovomucoid, cyclodextrins, HPLC, CE

Received 28 November 2017 / Accepted 28 December 2017

\section{Introduction}

During the past years, one of the main research direction in the pharmaceutical industry is the implications of chirality in the pharmacological effect and therapeutic use, respectively the development of modern enantioseparation methods for chiral drugs. Although high performance liquid chromatography (HPLC) remains a powerful technique in analysis of chiral drugs, capillary electrophoresis (CE) offers advantages in terms of low consumption of organic solvents and the wide variety of chiral selectors that can be easily added into background electrolyte solutions [1].

In the past years, chromatographic methods have been the first choice for the enantioseparation of chiral molecules. Nowadays, CE using cyclodextrins (CD) as chiral selectors presents many advantages compared to HPLC. Worth to mention that in CE, the consumption of organic solvents is relatively low; the type, ionic strength, concentration of electrolytes and the $\mathrm{pH}$ of the background electrolyte (BGE), can be easily changed; the buffers that can be used may be polar or non-polar. More importantly is that, in $\mathrm{CE}$, the chiral selector can be easily changed $[2,3]$. HPLC is a very well known technique offering high robustness and reproducibility. Nevertheless, in LC related chiral analysis methods, the chiral selector usually can be found at the level of the stationary phase. Thus beeing said, the versatility in changing the chiral selector resides

\footnotetext{
* Correspondence to: Alina Balint
}

E-mail: alina.balint@umftgm.ro in changing the chromatographic column, which involves high costs due to the high purchasing prices of chiral columns [4].

An interesting drug molecule with optical activity is amlodipine. Amlodipine (AML), (3-ethyl 5-methyl-2-[(2aminoethoxy)methyl]-4-(2-chlorophenyl)-6-methyl-1,4dihydropyridine- 3,5 dicarboxylate), a leader among dihydropyridine class, is used alone or in combination with other active pharmaceutical ingredients in various cardiovascular pathologies [5]. Amlodipine has one chiral center, due to the presence of an asymmetric carbon atom in position 4, which automatically generates two optical isomers (Figure 1). Amlodipine is available in pharmaceutical dosage forms as racemic mixture, although, the eutomer, levamlodipine (S-AML) was reported to be 1000 times more active than R-AML [6]. In few countries amlodipine is available as $S$ enantiomer tablets.<smiles>CCOCCOCc1c(C(=O)OCCN)[Y](-c2ccccc2Cl)c(C(=O)OC)c1C</smiles>

Fig. 1. Amlodipine chemical structure $\left(^{\star}\right.$ denotes the asymmetric carbon atoms) 
In recent years, only few HPLC chiral methods have been reported for the chiral discrimination of AML enantiomers, such as an enantioseparation with the aid of a Lux Cellulose- 4 chiral column by using a mobile phase composed of acetonitrile (ACN), ethanol and diethylamine [7]. Another HPLC method for amlodipine separation was developed by Luksa et al. [8]. They used $\alpha 1$ - acid glucoprotein immobilized on $5 \mu \mathrm{m}$ silica gel column and a mobile phase consisting of acetate buffer and propranol. The ovomucoid has been also used in the past by Ansari et al. [9] for its ability to distinguish between the enantiomers of many drugs. They developed an isocratic HPLC method for the enantiopurity quantitation of S-AML by using an OVM chiral column and a mobile phase consisted of phosphate buffer and acetonitrile.

In terms of chiral electrophoretic analytical methods developed for the enantioseparation of amlodipine, there are many methods reported in the literature where cyclodextrins were used as chiral selectors. Zandkarimi et al. [10] have succeeded the enantioseparation of amlodipine using highly sulfated cyclodextrins (HS- $\beta-C D$ ) as chiral selector. Another derivatized anionic cyclodextrin used by Owens et al. [11] aimed to solve the chiral separation of amlodipine was sulfobutylether- $\beta$-cyclodextrin (SBE- $\beta-C D)$. Neutral hydroxypropyl- $\beta-\mathrm{CD}$ (HP- $\beta-\mathrm{CD})$ were used by Wang et al. [12] for the enantioseparation of amlodipine. The use of randomly methylated $\beta$-CD (RAMEB- $\beta-C D$ ) as chiral selector by Hancu et al. [13] allowed the chiral separation of amlodipine with a resolution greater than 2. Native CD, $\alpha-C D$, has been used for AML enantiomers separation using CE by Small et al. [14] with the migration times longer than 25 minutes for both enantiomers.

Our study was focused on investigating the chiral enantioseparation behaviour of AML, with the help of two different newly developed methods. The chosen methods were based on HPLC and CE techniques, aiming in the separation of amlodipine enantiomers in less than $10 \mathrm{~min}$ utes analysis time.

\section{Materials and methods} Chemicals and reagents

Racemic amlodipine besylate and its pharmacologically active enantiomer, S-amlodipine used as reference substances were purchased from Sigma Aldrich (Germany). Acetonitrile $(\mathrm{ACN})$, methanol $(\mathrm{MeOH})$ and ethanol (EtOH) (LC Grade, Merck, Germany) were used as solvents in the composition of mobile phases or as solvents for standard solutions preparation. Sodium dihydrogen phosphate, disodium hydrogen phosphate, sodium hydroxide and orto-phosphoric acid (Merck, Germany) were used for the preparation of the HPLC mobile phases and for the ECZ buffers solutions. A Millipore Direct Q water purification system was used to obtain ultra-pure water. As chiral selectors for ECZ analysis various types of cyclodextrins were used: $\alpha$-cyclodextrin $(\alpha-C D), 2$-hydroxypropyl$\beta$-cyclodextrin (2 HP- $\beta$-CD), randomly methylated- $\beta$-cyclodextrin (RAMEB- $\beta$-CD), $\gamma$-cyclodextrin $(\gamma$-CD) (Cyclolab, Hungary).

\section{Instruments}

\section{Chromatographic conditions}

The HPLC stereoselective method of amlodipine was developed on an Agilent 1100 Series System (Agilent Technologies, USA) equipped with an UV-VIS detector. As chiral stationary phase, an Ultron ES OVM was used, packed in a $150 \times 4.6 \mathrm{~mm}$ column $(5 \mu \mathrm{m}$ particles $)$ (Shinwa Chemical Industries LTD, Agilent Technologies). The chiral selector consisted of ovomucoid glycoprotein immobilized on an aminopropylsilane-derivatized silica column. Different mobile phases were tested, containing different proportions of aqueous component as phosphate buffers (Na2HPO4; $\mathrm{NaH} 2 \mathrm{PO} 4)$ and various proportions of organic modifiers (ethanol, methanol and acetonitrile). The mobile phases were degassed by ultrasonication (Elma Transsonic bath) for 15 minutes prior to use.

\section{Electrophoretic conditions}

The CE enantioseparation of amlodipine besylate was performed on an Agilent Technologies 7100 CE equipped with a diode array UV detector. The equipment was assisted by ChemStation 7.01 software. The AML solutions were hydrodynamically injected at the anodic end of the capillary. Uncoated fused silica capillaries $(50 \mathrm{~cm}$ x $50 \mathrm{~mm}$ - Agilent, Germany) were used, while different types of cyclodextrins have been screened for stereospecific interactions with amlodipine: $\alpha-C D, 2-H P-\beta-C D$, RAMEB$\beta$-CD and $\gamma$-CD. BGEs were prepared by dissolving the appropriate amount of buffer (H3PO4; Na2HPO4; $\mathrm{NaH}-$ $2 \mathrm{PO} 4)$ in ultrapure water and adjusting the $\mathrm{pH}$, when it was necessary, with $\mathrm{NaOH} 1 \mathrm{M}$ or $\mathrm{H} 3 \mathrm{PO} 410 \%$. The capillary was conditioned with $\mathrm{NaOH} 0.1 \mathrm{M}$ for 30 minutes, followed by purified water for 15 minutes and finally with the BGE for 15 minutes. Between runs, the capillary was preconditioned with purified water for one minute and with the BGE, for two minutes.

The detection undertook for both methods at the wavelength value of $238 \mathrm{~nm}$, where amlodipine recorded maximum absorbance. The Terminal 740 (Inolab) pH meter, previously calibrated, was used for $\mathrm{pH}$ buffer adjustments of mobile phases (HPLC) and for BGE solutions (CE). An ultrasonic bath T700H (Elma Transsonic) was used for the preparation and degassing of the mobile phases.

\section{Samples, mobile phases and BGE preparations}

AML stock solutions were prepared by weighing a suitable amount of powder and disolving it in methanol in order to give a concentration level of $1000 \mu \mathrm{g} / \mathrm{mL}$ in a volumetric flask. Stock solutions were later diluted to the appropriate concentration with the mobile phase mixture. S-AML stock solution was prepared also in methanol, at a concentration level of $10 \mu \mathrm{g} / \mathrm{mL}$. CDs solutions were prepared at a concentration level of $50 \mathrm{mM}$ by dissolving the $\mathrm{CD}$ in 
BGE. All samples, CDs and buffer solutions, were filtered through a $0.45 \mu \mathrm{m}$ syringe filter and ultrasonicated for five minutes prior to use. The detection took place at the cathode end of the capillary.

\section{Results and discussions HPLC method development and optimization}

The proposed HPLC method is based on the separation due to the interaction with chiral stationary phase CSP. The chiral selector belongs to the protein class, chemically bonded to spherical silica gel particles. The ovomucoid, a chiral polymer is able to present stereoselective interactions with a large number of pharmacologically active compounds, exhibiting a very complex recognition mechanism. The chiral selector contains chiral recognition sites for a pair of enantiomers, and the chiral separation mechanisms are based on unique combinations of hydrophobic and polar interactions between the chiral analyte and ovomucoid molecules [15].

Since the overall charges both for the ionizable enantiomers and the proteic stationary phases are influenced by the $\mathrm{pH}$, one of the most important parameters for enantiomeric separation on an ovomucoid is the $\mathrm{pH}$ of the mobile phase by taking in consideration to the $\mathrm{pKa}$ of the analyte $(\mathrm{pKaAML}=9.10)$.

OVM has an isoelectric point $(\mathrm{pI})$ of 4.1, therefore, it is expected for the stationary phase to be negatively charged when the mobile phase $\mathrm{pH}$ is above the $\mathrm{pI}$ values. The decrease of the mobile phase $\mathrm{pH}$ towards the ovomucoid $\mathrm{pI}$ reduces the negative charges of the stationary phase, resulting in shorter retention for the basic compounds, whereas increasing in the mobile phase $\mathrm{pH}$ towards the $\mathrm{pI}$ reduces the positive charge of the stationary phase, resulting thus in shorter retention times for acidic compounds $[15,16]$.

Taking into account these facts, the HPLC method was developed and improved by changing some of the critical chromatographic conditions such as the $\mathrm{pH}$ of the mobile phase's aqueous component, proportion of the mobile phase's organic modifier (ethanol, methanol or acetonitrile), the chromatographic column temperature, all in an univariate manner.

The influence of the mobile phase's aqueous component $\mathrm{pH}$ was studied at different $\mathrm{pH}$ values between 3 and 7 . It is worth mentioning that the tested $\mathrm{pH}$ interval fell within the manufacturer recommended $\mathrm{pH}$ usage range of the column.

The optimal $\mathrm{pH}$ value with whom the chiral resolution and selectivity are associated with enantioseparation of amlodipine, is $5.0(\mathrm{R}=5.51 ; \alpha=1.71)$. The obtained retention times were shorter than 10 minutes for the two isomers, $\mathrm{tR}(\mathrm{S}-\mathrm{AML})=4.90(\mathrm{~min}) ; \mathrm{tR}(\mathrm{R}-\mathrm{AML})=7.18$ (min). (Figure 2)

Taking into account that the temperature has an important role on all chromatographic techniques and due to the fact that both the thermodynamics and kinetics of

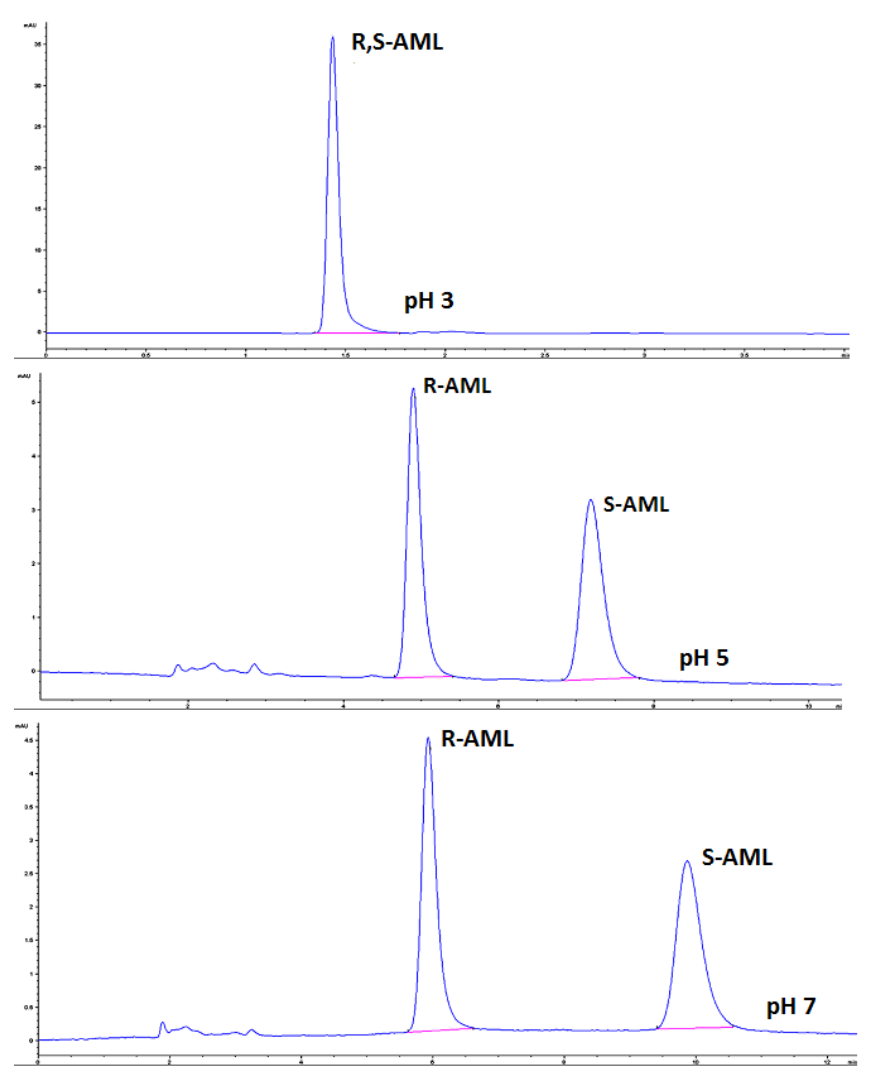

Fig. 2. Chromatograms of the enantioseparation for racemic AML at different $\mathrm{pH}$ values $(50 \mu \mathrm{g} / \mathrm{ml})(\mathrm{HPLC}$ conditions: Ultron ES-OVM column; $25^{\circ} \mathrm{C}$; mobile phase: $\mathrm{A}-\mathrm{Na}_{2} \mathrm{HPO}_{4} 10 \mathrm{mM} \mathrm{78 \% ,} \mathrm{B} \mathrm{-} \mathrm{ACN}$ $22 \%$, flow $1 \mathrm{ml} / \mathrm{min}$, isocratic elution).

adsorption processes are temperature dependant, column temperature was varied between $20-35^{\circ} \mathrm{C}$. This temperature range is supported by the stationary phase, according to the producer statement. In this study, lowering the elution temperature produces a decrease in retention time and an improving, consecutively, of the peaks shape. Elevated temperatures are able to decrease the viscosity of the mobile phase and to increase solubility and diffusivity, improving thus the separation resolution and the selectivity of the method. (Table I)

Optimal HPLC experimental conditions were established at a column temperature of $30^{\circ} \mathrm{C}$, the percentage of the aqueous buffer component of the mobile phase was $80 \%(\mathrm{Na} 2 \mathrm{HPO} 410 \mathrm{mM})$ at a $\mathrm{pH}$ level of 5.0 . The organic modifier, consisting of $\mathrm{ACN}$, turned to be optimal when it is used under a proportion of $20 \%$. The elution mode was isocratic at a flow of $1 \mathrm{ml} / \mathrm{min}$. The established chromatographic conditions allowed the separation of the enantiomers of amlodipine, in less than 7 minutes.

Table I. Variation of separation parameters $\left(t_{R}, N, R, \alpha\right)$ of amlodipine depending on the elution temperature

\begin{tabular}{lcccccc}
\hline \multirow{2}{*}{ Column temperature } & \multicolumn{7}{c}{ Amlodipine } \\
& $\mathrm{t}_{\mathrm{R} 1}$ & $\mathrm{t}_{\mathrm{R} 2}$ & $\mathrm{~N}_{1}$ & $\mathrm{~N}_{2}$ & $\mathrm{R}_{\mathrm{s}}$ & $\alpha$ \\
\hline $20^{\circ} \mathrm{C}$ & 5.02 & 7.66 & 3706 & 2740 & 5.76 & 1.79 \\
$25^{\circ} \mathrm{C}$ & 4.89 & 7.18 & 3801 & 3157 & 5.51 & 1.71 \\
$30^{\circ} \mathrm{C}$ & 4.63 & 5.54 & 3894 & 3263 & 5.04 & 1.65 \\
$35^{\circ} \mathrm{C}$ & 4.57 & 6.01 & 4248 & 3688 & 4.26 & 1.50 \\
\hline
\end{tabular}




\section{CE method development and optimization}

The selectivity for capillary electrophoresis depends primarily on the electrical charge, which can be induced by changing the $\mathrm{pH}$ of the buffer solution and the ionic strength. The buffer composition and the ionic strength can influence the enantiorecognition, because the mobility of the analytes and cyclodextrins, respectively, are strongly affected. In the case of CE, cyclodextrins play the role of chiral selectors, the separation of chiral molecules can be achieved in CE, given the fact that it forms inclusion complexes with different stability and mobilities for the two isomers $[2,3]$.

To improve the selectivity in CE, using cyclodextrins as chiral selectors, the optimum buffer concentration was determined by recording the electropherograms of racemic amlodipine, under identical conditions, at different concentrations of the phosphate buffer: $25 \mathrm{mM}, 50 \mathrm{mM}, 75$ $\mathrm{mM}$ or $100 \mathrm{mM}$. High separation selectivity was observed when using the most diluted phosphate buffer solution, $25 \mathrm{mM}$.

The $\mathrm{pH}$ of the BGE also turned to be a critical parameter. This happened due to the fact that it influenced the electric charge of both the analyte and the cyclodextrin, modifying thus the electrostatic interactions between the chiral selector and the two isomers, in the end resulting in the different mobility of the complexes. Considering the pKa value of amlodipine (9.1) the electropherograms have been recorded in a $\mathrm{pH}$ range comprised between 2.0 to 7.0. The best stereoresolution of the amlodipine enantiomers was achieved at a $\mathrm{pH}$ value of 3.0.

To improve the selectivity CE, the optimum BGE concentration was determined by recording the electropherograms of racemic AML, using different concentrations of the phosphate buffer between 25-100 mM. High separation selectivity was observed when using $25 \mathrm{mM}$ phosphate buffer solutions.

Once the optimum composition and concentration of the BGE had been established, the enantiomeric resolution of AML was further studied using several types of native $C D: \alpha-C D ; \beta-C D$ and $\gamma-C D$ and derivatized $\beta-C D$ : 2 -HP- $\beta$-CD, RAMEB- $\beta$-CD. The CDs were added in different concentrations relative to the used buffers in order to observe the enantiomeric separation mechanism, taking in consideration chiral resolutions. (Figure 3)

According to the Dalgliesh's three-point interaction rule, chiral recognition is possible in a context where at least three simultaneous interactions between the enantiomer and the used chiral selector must exist.

Although AML is a relatively large molecule presenting two aromatic rings in its chemical structure, the $\mathrm{CD}$ with the smallest inner cavity, $\alpha$-CD was the only one able to support the complete separation under the electrophoretic conditions mentioned above. Regarding the AML molecule from 3D perspective with molecular energy optimization the 2 aromatic rings are almost orthogonal one with respect to the other and the benzene ring has no steric
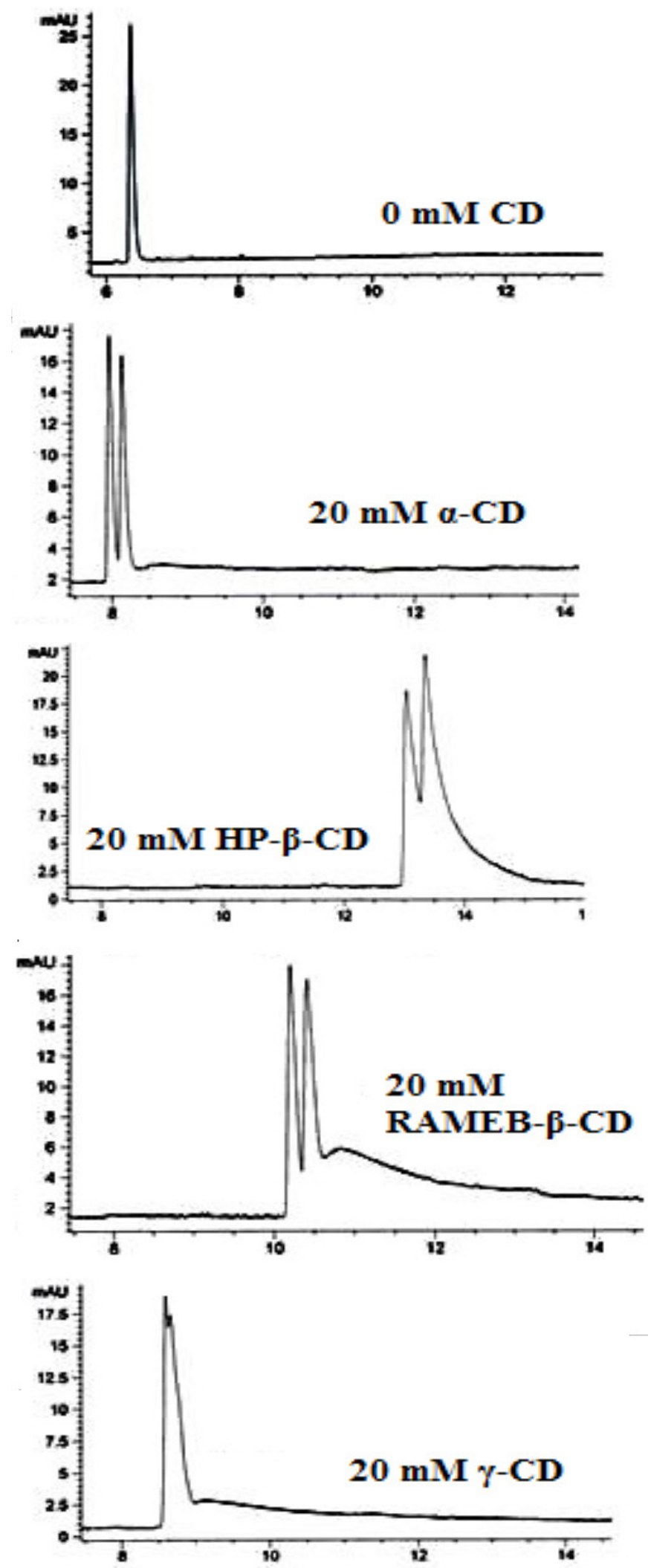

Fig. 3. Electropherograms of the chiral separation for racemic $A M L$ using different $\mathrm{CD}$ as chiral selectors (CE conditions: phosphate buffer $25 \mathrm{mM}, \mathrm{pH} 3$, injection pressure/time: $30 \mathrm{mbar} / 3 \mathrm{sec}$, voltage $+25 \mathrm{kv}, 25^{\circ} \mathrm{C}$, CD concentration $20 \mathrm{mM}$ : a) $\alpha \mathrm{CD}$; b) $2 \mathrm{HP}-\beta-\mathrm{CD}$; c) RAMEB- $\beta-C D$; d) $\gamma$-CD).

hindrance in the S-enantiomer, therefore a higher binding energy between S-AML and cyclodextrin is more probably. [3] 
After the establishment of the optimum chiral selector, the capillary temperature was varied between $15^{\circ} \mathrm{C}$ and $30^{\circ} \mathrm{C}$. This was done only to observe if the capillary temperature in the case of electrophoretic determination may represent a critical parameter as in the case of chromatographic determination.

The increase in capillary temperature leads to a decreased viscosity of the buffer solution, altering both the chiral AML and CD-analyte complex mobility. Whereas the chromatographic method was negatively influenced by the increase of the temperature as both the resolution and the selectivity were lowered, in the case of the electrophoretic method, the variation of capillary temperature affects only the resolution, without affecting the enatiomeric selectivity. (Table II)

The optimum CE conditions seemed to be based on using a buffer solution containing $25 \mathrm{mM} \mathrm{H} 3 \mathrm{PO} 4$ at $\mathrm{pH} 3.0$ and $20 \mathrm{mM} \alpha-\mathrm{CD}$ as chiral selector. The optimum separation underwent under an applied voltage of $+25 \mathrm{kV}$, a capillary temperature set at $15^{\circ} \mathrm{C}$ and UV detection at 238 $\mathrm{nm}$. Under these parameters, the separation for the two enantiomers of amlodipine succeeded in approximately 10 minutes, with a resolution of 1.51 and a stereoselectivity factor of 1.03. (Figure 4)

The elution order and the migration time of the enantiomers were established by injecting the solution of the pure enantiomer, S-AML, in the same optimiezed chromatographic/electrophoretic conditions, and was S-AML followed by R-AML.

Table II Electrophoretic parameters $(R, \alpha)$ recorded following of enantioseparation of amlodipine

\begin{tabular}{ccccccc}
\hline $\begin{array}{c}\text { Capillary } \\
\text { temperature }\end{array}$ & \multicolumn{2}{c}{$\alpha-\mathrm{CD}$} & \multicolumn{2}{c}{ 2 HP- $\beta$-CD } & \multicolumn{2}{c}{ RAMEB- $\beta-\mathrm{CD}$} \\
& $\mathrm{R}$ & $\alpha$ & $\mathrm{R}$ & $\alpha$ & $\mathrm{R}$ & $\alpha$ \\
\hline $15^{\circ} \mathrm{C}$ & 1.51 & 1.03 & 1.05 & 1.02 & 0.94 & 1.02 \\
$18^{\circ} \mathrm{C}$ & 1.46 & 1.03 & 1.04 & 1.02 & 0.91 & 1.02 \\
$21^{\circ} \mathrm{C}$ & 1.32 & 1.03 & 1.01 & 1.02 & 0.90 & 1.02 \\
$24^{\circ} \mathrm{C}$ & 1.25 & 1.03 & 0.96 & 1.02 & 0.89 & 1.02 \\
$27^{\circ} \mathrm{C}$ & 1.22 & 1.03 & 0.72 & 1.02 & 0.86 & 1.02 \\
$30^{\circ} \mathrm{C}$ & 1.09 & 1.03 & 0.69 & 1.02 & 0.84 & 1.02 \\
\hline
\end{tabular}

Table IV Analytical performance for the proposed HPLC and CE methods

\begin{tabular}{lcccccc}
\hline Enantiomer & \multicolumn{3}{c}{ HPLC } & & \multicolumn{3}{c}{ CE } \\
& $\begin{array}{l}\text { Retention } \\
\text { time (min) }\end{array}$ & $\mathrm{R}$ & $\alpha$ & $\begin{array}{c}\text { Migration } \\
\text { time (min) }\end{array}$ & $\mathrm{R}$ & $\alpha$ \\
\hline R-AML & 4.63 & 5.04 & 1.65 & 8.15 & 1.51 & 1.03 \\
S-AML & 5.54 & & & 8.45 & & \\
\hline
\end{tabular}

The evaluation of the analytical performances of the two proposed chiral methods

Calibrations of the two methods were achived by fitting a linear curve to five concentration points covering ranges between 20-500 $\mu \mathrm{g} / \mathrm{mL}$ for AML enantiomers (HPLC method) and 50-500 $\mu \mathrm{g} / \mathrm{mL}$ (CE method), respectively. Three independent injections were performed at each concentration level.

The limit of detection (LOD) for the two isomers was established in terms of peak height to background noise signal ratio, which must not be less than three times the noise signal intensity for both of the proposed methods. The limit of quantification (LOQ) was set at the lowest concentration at which the peak's height of the analytes were 10 times more intense than the noise signal intensity. As can be seen, the limits of detection and the limits of quantification for the two developed methods are substantially different. (Table III and IV)

The two proposed methods were statistically evaluated by comparing the variances of the retention times ( $\mathrm{tR}$ HPLC) and migration times (tm-CE) of the amlodipine enantiomers ( $F$ test, $N=5, \alpha=0.05$ ). (Table $V$ )

$F$ test revealed that, there is no statistical difference between the variances of the retention times obtained with the help of HPLC method compared to the variances of the migration times obtained when using the CE method, for both enantiomers, R-AML and S-AML, respectively.

\section{Conclusions}

Both developed methods turned to comply to the desired separation performance parameters in terms of resolution, selectivity and migration times. Analysis time is was short-

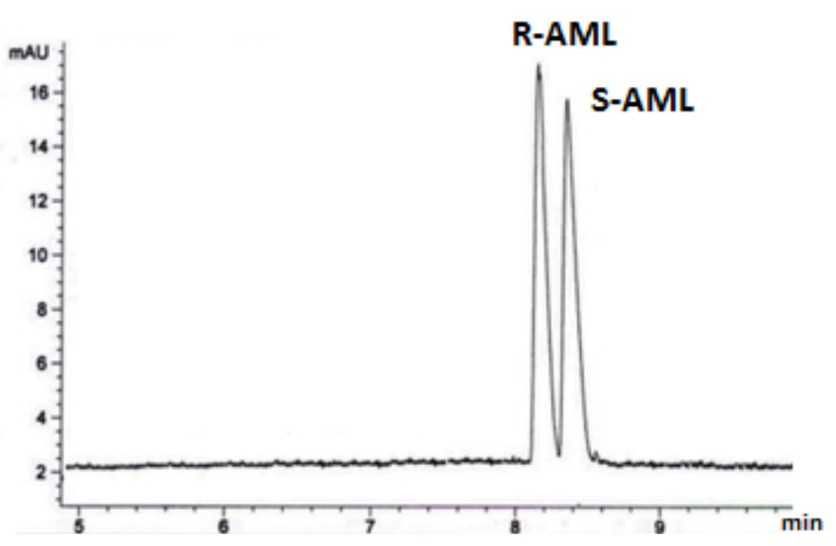

Fig. 4. Typical electropherogram for racemic amlodipine $(100 \mu \mathrm{g} /$ $\mathrm{ml}$ ) (analytical conditions: $25 \mathrm{mM}$ phosphate buffer, $\mathrm{pH} 3.0,20 \mathrm{mM}$ $\alpha-C D$ chiral selector, voltage $+25 \mathrm{kV}$, temperature $15^{\circ} \mathrm{C}$, injection pressure/time: $30 \mathrm{mbar} / 3 \mathrm{sec}$, UV detection $238 \mathrm{~nm}$ )

Table III The limit of detection (LOD) and limit of quantitation (LOQ) for the proposed HPLC and CE chiral method

\begin{tabular}{|c|c|c|c|c|c|c|c|c|}
\hline \multirow[t]{2}{*}{ Enantiomer } & \multicolumn{6}{|c|}{ HPLC } & \multicolumn{2}{|c|}{ CE } \\
\hline & Regression equation & $\mathrm{R}^{2}$ & $\begin{array}{l}\text { LOD } \\
(\mu \mathrm{g} / \mathrm{ml})\end{array}$ & $\begin{array}{l}\text { LOQ } \\
(\mu \mathrm{g} / \mathrm{ml})\end{array}$ & $\begin{array}{l}\text { Regression equa- } \\
\text { tion }\end{array}$ & $\mathrm{R}^{2}$ & $\begin{array}{l}\text { LOD } \\
(\mu \mathrm{g} / \mathrm{ml})\end{array}$ & $\begin{array}{l}\text { LOQ } \\
(\mu \mathrm{g} / \mathrm{ml})\end{array}$ \\
\hline R-AML & $y=4.835 x-31.34$ & 0.999 & 2.02 & 6.31 & $y=9.016 x-29.69$ & 0.998 & 10.06 & 29.69 \\
\hline S-AML & $y=4.829 x-31.83$ & 0.999 & 2.12 & 6.43 & $y=9.013 x-31.58$ & 0.997 & 10.33 & 30.67 \\
\hline
\end{tabular}


Table V Comparison between qualitative precision of the two methods, HPLC and CE

\begin{tabular}{|c|c|c|c|c|c|}
\hline $\begin{array}{l}\text { R-AML } \\
\text { HPLC }\left(t_{R}\right) \text { vs CE }\left(t_{m}\right)\end{array}$ & Variable 1 & Variable 2 & $\begin{array}{l}\text { S-AML } \\
\operatorname{HPLC}\left(\mathrm{t}_{\mathrm{R}}\right) \text { vs CE }\left(\mathrm{t}_{\mathrm{m}}\right)\end{array}$ & Variable 1 & Variable 2 \\
\hline Mean & 4.592 & 8.282 & Mean & 5.484 & 8.492 \\
\hline Variance & 0.00082 & 0.00772 & Variance & 0.00328 & 0.00152 \\
\hline CV\% & 0.6236 & 1.0443 & CV\% & 1.0609 & 0.4591 \\
\hline Observations & 5 & 5 & Observations & 5 & 5 \\
\hline $\mathrm{F}$ & \multicolumn{2}{|c|}{0.1062} & $\mathrm{~F}$ & \multicolumn{2}{|c|}{2.1579} \\
\hline$P(F<=f)$ one-tail & \multicolumn{2}{|c|}{0.0517} & $P(F<=f)$ one-tail & \multicolumn{2}{|c|}{0.4745} \\
\hline F Critical one-tail & \multicolumn{2}{|c|}{0.1565} & F Critical one-tail & \multicolumn{2}{|c|}{6.3882} \\
\hline
\end{tabular}

er than 7 minutes for the HPLC method and shorter than 10 minutes in the case of CE method.

The analytical performances of the chromatographic method using OVM as chiral selector (R-5.04; $\alpha-1.71$; $\mathrm{LOD}-2 \mu \mathrm{g} / \mathrm{mL}$ ) are superior to the electrophoretic analysis method (R-1.51; $\alpha-1.03$; LOD $-10 \mu \mathrm{g} / \mathrm{mL}$ ). Although analysis times from the literature published in previous studies are similar with those obtained in this study, the enantioresolution of our method $(\mathrm{R}=5.04)$ is clearly superior $(\mathrm{R}=1.43)$.

The proposed electrophoretic method, using $\alpha-\mathrm{CD}$ as chiral selector to the BGE, has been successfully applied for the steric discrimination of AML enantiomers, with good repeatability of the migration time and peaks areas. More to be added, the method is more economical may represent an alternative to the HPLC chromatographic separation.

Although the evaluation of the analytical performances of the two methods indicates that the HPLC method is more efficient in enantioseparation of AML, due to low solvent, analyte and chiral selector consumption and lower operational cost, the CE proposed method represents a viable alternative for the quantitative determination of $\mathrm{AML}$ emantiomers in pharmaceutical forms.

\section{Conflict of interest}

None to declare.

\section{Acknowledgement}

This paper is supported with a project funded through Internal Research Grants by the University of Medicine and Pharmacy of Tîrgu Mureş, Romania (grant contract for execution of research projects nr. 17800/4/22.12.2015).

\section{References}

1. Zhang Y, Yao S, Zeng H, Song H - Chiral Separation of Pharmaceuticals by High Performance Liquid Chromatography. Curr Pharm Anal. 2010;6:114-130.

2. Saz JM, Marina ML - Recent advances on the use of cyclodextrins in the chiral analysis of drugs by capillary electrophoresis, J Chromatogr
A. 2016;1467:79-94.

3. Al Azzam KM, Abdallah HH, Abdul Halim HN, Ahmad MA, Shaibah $\mathrm{H}$ - Host-Guest Inclusion Complexes between Amlodipine Enantiomers in the Biphasic Recognition Chiral Extraction System using Tartaric Acid and ?-Cyclodextrin Derivatives as Positive Confirmation by using their Enantioselective Extraction. Sci Pharm 2015;83(4):683-698.

4. Bi C, Zheng X, Azaria S, Beeram S, Li Z, Hage DS - Chromatographic Studies of Protein-Based Chiral Separations. Separations 2016;3(3):27.

5. Arrowsmith JE, Campbell SF, Cross PE, Stubbs JK, Burges RA Gardiner DG. et al. - Long-acting dihydropyridine calcium antagonists. 1. 2-Alkoxymethyl derivatives incorporating basic substituents. J Med Chem. 1986;29(9):1696-1702.

6. Hotha KK, Roychowdhury S, Mullangi R, Ravindranath LK - Rapid quantification of amlodipine enantiomers in human plasma by LCMS/MS: Application to a clinical pharmacokinetic study. Biomed Chromatogr. 2013,27:1192-1199.

7. Kannappan V, Mannemala SS - Simultaneous enantioseparation and purity determination of chiral switches of amlodipine and atenolol by liquid chromatography. J Pharm Biomed Anal. 2016; 120:221-227.

8. Luksa J, Josíc D, Podobnik B, Furlan B, Kremser M - Semi-preparative chromatographic purification of the enantiomers S-(-)-amlodipine and R-(+)-amlodipine. J Chromatogr B Biomed Sci Appl. 1997;693(2):367375.

9. Ansari KA, Pagar KP, Vavia PR - Development and validation of HPLC method for the determination of S (-) Amlodipine Besylate and its related substance in tablet formulation by using chiral separation. Asian $J$ Biomed Pharm Sci. 2012;2(12):60-64.

10. Zandkarimi M, Shafaati A, Foroutan SM, Lucy CA - Rapid enantioseparation of amlodipine by highly sulfated cyclodextrins using short-end injection capillary electrophoresis. DARU. 2009;17(4):269275.

11. Owens PK, Fell AF, Coleman MW, Berridge JC - Effect of charged and uncharged chiral additives on the resolution of amlodipine enantiomers in liquid chromatography and capillary electrophoresis. J Chromatogr A. 1998;797:187-195.

12. Wang R, Jia Z, Fan JJ, Chen LR, Xie H, Ma J, Ge X, Zhang Q, Ao Y, Wang $J$ - CE, with hydroxypropyl- $\beta$-cyclodextrin as chiral selector, for separation and determination of the enantiomers of amlodipine in the serum of hypertension patients. Chromatographia. 2007;65:575-579.

13. Hancu G, Budău M, Kántor LK, Cârje A, Cyclodextrin screening for the chiral separation of amlodipine enantiomers by capillary electrophoresis. Adv Pharm Bull. 2015;5(1):35-40.

14. Small SS, Fell AF, Coleman MW, Berridge JC - Central composite of ruggedness and design for the rapid optimisation chiral separation of amlodipine in capillary electrophoresis. Chirality. 1995;7:226-234.

15. Hermansson $\mathrm{J}$ - Direct liquid chromatographic resolution of racemic drugs by means of $\alpha 1$-acid glycoprotein as the chiral complexing agent in the mobile phase. J Chromatogr A. 1984; 316:537-546.

16. Oda Y, Mano N, Asakawa N, Yoshid Y, Sato T, Nakagawa T Comparison of Avidin and Ovomucoid as Chiral Selectors Resolution of Drug Enantiomers by High-Performance Liquid Chromatography. Anal Sci. 1993;9:221-228. 